\title{
Correlation of histopathologic features of ductal carcinoma in situ of the breast with the oncotype DX DCIS score
}

\author{
Adriana Knopfelmacher ${ }^{1}$, Jana Fox ${ }^{2}$, Yungtai Lo ${ }^{3}$, Nella Shapiro ${ }^{4,5}$ and Susan Fineberg ${ }^{1}$ \\ ${ }^{1}$ Department of Anatomic Pathology, Montefiore Medical Center and the Albert Einstein College of Medicine, \\ Bronx, NY, USA; ${ }^{2}$ Department of Radiation Oncology, Montefiore Medical Center and the Albert Einstein \\ College of Medicine, Bronx, NY, USA; ${ }^{3}$ Department of Epidemiology and Population Health and Department \\ of Pathology, Montefiore Medical Center and the Albert Einstein College of Medicine, Bronx, NY, USA; \\ ${ }^{4}$ Department of Surgery, Montefiore Medical Center and the Albert Einstein College of Medicine, Bronx, NY, \\ USA and ${ }^{5}$ Eastchester Center for Cancer Care, Bronx, NY, USA
}

\begin{abstract}
The Oncotype DX Breast Cancer Assay for ductal carcinoma in situ is used to determine local recurrence risk in patients with ductal carcinoma in situ. The results help select patients with low-risk ductal carcinoma in situ who could forgo radiation therapy after conservative surgery. The genes assessed include five proliferation genes, progesterone receptor (PR), and GSTM-1. Our objective was to determine if PR, mitotic counting, or any other pathologic feature of ductal carcinoma in situ could predict the Oncotype DX DCIS Score. We identified 46 cases of ductal carcinoma in situ with a Oncotype DX DCIS Score. In addition to information obtained from routine pathology, we counted mitotic figures in the ductal carcinoma in situ and noted presence of dense chronic inflammatory infiltrate surrounding ductal carcinoma in situ. We found that $P R \geq 90 \%(P=0.004)$, mitotic count $\leq 1(P=0.045)$, estrogen receptor $\geq 90 \%(P=0.046)$, and low nuclear grade $(P<0.0001)$ were associated with a low score. Dense chronic inflammation surrounding ductal carcinoma in situ was associated with a high score $(P=0.034)$.All 13 cases with $P R \geq 90 \%, \leq 1$ mitotic figure and absence of dense chronic inflammation around ductal carcinoma in situ had a low score ( $100 \%$ specificity). A low score was not observed in any case with at least two of the following-negative PR, $>1$ mitotic figure, and/or presence of dense chronic inflammation around ductal carcinoma in situ (100\% specificity). Our study suggests using a combination of PR ( $\geq 90 \%$ vs negative) with mitotic count in ductal carcinoma in situ ( $\leq 1$ vs $>1)$ and dense chronic inflammation around ductal carcinoma in situ one could predict the Oncotype DX DCIS score. Mitotic counting and evaluation of immune response might provide prognostic information in ductal carcinoma in situ.

Modern Pathology (2015) 28, 1167-1173; doi:10.1038/modpathol.2015.79; published online 26 June 2015
\end{abstract}

The majority of women in the United States with ductal carcinoma in situ (DCIS) of the breast are treated with breast conserving surgery. ${ }^{1}$ The risk of local recurrence after breast conserving surgery alone is up to $30 \%$ and half of the recurrences are invasive breast cancer. ${ }^{2,3}$ The addition of radiotherapy has been shown to decrease local recurrence risk by about $50 \%$; hence, the majority of patients

Correspondence: Dr S Fineberg, MD, Department of Anatomic Patholgy, Montefiore Medical Center and the Albert Einstein College of Medicine, 4th floor Silver Zone, 111 East 210th Street, Bronx, NY 10467, USA.

E-mail sfinebergshear@hotmail.com

Received 14 February 2015; revised 11 May 2015; accepted 12 May 2015; published online 26 June 2015 will receive radiotherapy after conservative breast surgery for DCIS., ${ }^{4,5}$

The risk of local recurrence after breast conserving surgery for DCIS has been shown to be related to presence of high-grade DCIS, close or involved margins, age under 50, and larger tumor size., ${ }^{3,5,6}$ Studies have attempted to identify patients with lowrisk DCIS who could forgo radiation therapy. One study found that patients with low- or intermediategrade DCIS measuring $2.5 \mathrm{~cm}$ or less and with a margin of $3 \mathrm{~mm}$ or greater have an acceptable low rate of breast recurrence of $6.1 \%$ at 5 years and are a group where radiation might be omitted. ${ }^{7}$ However, even in this low-risk group, there remains a benefit from radiation therapy. ${ }^{8}$ Thus, well-defined criteria to select patients for surgical excision alone without radiation therapy for DCIS have not yet firmly been 
established. As radiation therapy has no overall impact on survival and has associated costs and morbidity, more accurate determination of the patients least likely to benefit would be critical to avoid overtreatment. ${ }^{9}$

Genomic Health is a commercial laboratory that has developed a multigene reverse transcriptase (RT)-PCR assay designed to quantify the 10-year risk of local recurrence, both in situ and invasive, in patients with DCIS treated with breast conserving surgery without radiation. This assay, called the Oncotype DX Breast Cancer Assay for DCIS, provides information on local recurrence, which is independent of clinicopathologic features. The results are reported as a numerical score called the DCIS Score and divided into low-, intermediate-, and high-risk groups with overall local recurrence rates of 10.6, 26.7 , and $29.5 \%$, respectively, at 10 years. Invasive recurrence rates are $3.7,12.3$, and $19.2 \%$ for these groups, respectively. ${ }^{10}$ The score is intended to help select patients with low-risk DCIS that could potentially forgo radiation therapy.

The Oncotype DX Breast Cancer Assay for DCIS has several limitations. First, it is applicable only to a minority of patients who are considered low risk to begin with. These patients must have resection margins of at least $3 \mathrm{~mm}$ and have either low- or intermediate-grade DCIS measuring $2.5 \mathrm{~cm}$ or less, or if high-grade DCIS must be $1 \mathrm{~cm}$ or less in size, as this is the set of patients from ECOG 5194 study upon which the test was initially clinically validated. ${ }^{10}$ Second, the results of a recent large study brings into question the ability of the DCIS Score to differentiate intermediate- and high-risk DCIS, showing similar recurrence risks for both invasive and in situ ductal cancer when comparing intermediate and high DCIS Scores (33 vs $27.8 \%$ ). ${ }^{11}$ In addition, the cost of the test is about $\$ 4000.00$.

The Oncotype DX DCIS Assay includes assessment of seven prognostic genes including five proliferation genes, progesterone receptor, and GSTM1. Progesterone receptor is routinely assessed via immunohistochemistry in the pathologic assessment of all DCIS. Although formal assessment of proliferation is not routinely done for DCIS, mitotic figure counting could easily be done in cases of DCIS. GSTM1 is an enzyme whose function includes metabolism of environmental carcinogens, and loss of function is associated with increased cancer risk. ${ }^{12}$ GSTM1 is not routinely assessed in pathologic evaluation of DCIS. Thus two of the three categories of prognosticators evaluated in the Oncotype DX DCIS Assay can be evaluated by pathologic assessment.

In this study we reviewed the pathology of 46 cases of DCIS for which an Oncotype DX DCIS Breast Cancer Assay had been performed. We sought to determine if progesterone receptor evaluation, mitotic counting, or any other factors that could be evaluated by pathologic assessment could predict the Oncotype DX DCIS Score.

\section{Materials and methods}

Forty-six cases of DCIS were identified and sent from our institution to Genomic Health for an Oncotype DX Breast Cancer Assay for DCIS Score. All cases were from surgical pathology specimens received and reviewed by our Department of Pathology. Although a surgical resection specimen was reviewed in pathology in all cases, the Oncotype DX DCIS Assay was performed on the specimen with the greatest volume of DCIS, which could be either the diagnostic core biopsy or the surgical excision. Appropriate slides for Oncotype DX testing were selected by a pathologist.

In 35 of the 46 cases the hematoxylin and eosinstained slides from the block sent for Oncotype DX testing was available and reviewed for this study. In the remaining 11 cases the representative hematoxylin and eosin-stained slide was not in the file at the time of this review. In each case we counted the total number of mitotic figures in all glands with DCIS. We also noted if there was a dense chronic inflammatory infiltrate around DCIS, defined as circumferential or near circumferential $(>75 \%$ of circumference) cuffing of duct by lymphocytes or plasma cells at least three cell layers in thickness. Additional pathologic features of DCIS, which are routinely recorded in the pathology reports, including size, nuclear grade, presence of comedo necrosis, and margin status, were obtained from the pathology reports. Hormone receptor results for estrogen receptor (clone SP1 Cell Marque, Rocklin, CA) and progesterone receptor (clone 636 DAKO, Carpinteria, CA) were abstracted from pathology reports. As per the College of American Pathologists guidelines, positive hormone receptor staining was staining of $1 \%$ or greater of tumor nuclei. In addition to reporting positive or negative the estimated percentage of positive staining nuclei was reported. Estrogen or progesterone receptor positivity in $90 \%$ or greater staining of tumor nuclei was specifically noted.

The Oncotype DX DCIS Score was classified as low if $<39$, intermediate if $39-54$ and high if 55 or greater as per Oncotype DX DCIS Breast Cancer Assay guidelines.

Additional information abstracted from the patients' electronic medical record included patient age and whether radiation therapy was performed after conservative surgery for DCIS.

This study was approved by the institutional review board of our hospital.

\section{Statistical Analysis}

Differences in age and tumor size between low Oncotype DX DCIS Score, intermediate Oncotype DX DCIS Score, and high Oncotype DX DCIS Score were compared using ANOVA or Kruskal-Wallis tests when appropriate. Differences in mitotic 
Table 1 Associations between histopathologic features of DCIS and Oncotype DX DCIS Score

\begin{tabular}{|c|c|c|c|c|}
\hline & $\begin{array}{l}\text { Low oncotype DX DCIS } \\
\text { Score }(\mathrm{N}=33)\end{array}$ & $\begin{array}{c}\text { Intermediate oncotype } D X \\
\text { DCIS Score }(\mathrm{N}=8)\end{array}$ & $\begin{array}{l}\text { High oncotype DX } \\
\text { DCIS Score }(\mathrm{N}=4)\end{array}$ & $\mathrm{P}$ value \\
\hline Age, mean \pm s.d. & $64+10$ & $70+9$ & $53 \pm 6$ & 0.027 \\
\hline Tumor size (mm), median (range) & $6.0(2,31)$ & $9.0(3,70)$ & $5(2,10.0)$ & 0.193 \\
\hline Grade, N (\%) & & & & $<0.0001$ \\
\hline $\mathrm{I}$ & $7(21)$ & 0 & 0 & \\
\hline II & $26(79)$ & 3 (38) & $3(75)$ & \\
\hline III & 0 & $5(63)$ & $1(25)$ & \\
\hline Mitotic count, N (\%) & & & & 0.045 \\
\hline$\leq 1$ & $17(74)$ & $2(27)$ & $1(25)$ & \\
\hline$>1$ & $6(26)$ & $5(71)$ & $3(75)$ & \\
\hline Comedo necrosis, $\mathrm{N}(\%)$ & & & & 0.070 \\
\hline Yes & $10(30)$ & $6(75)$ & $1(25)$ & \\
\hline No & $23(70)$ & $2(25)$ & $3(75)$ & \\
\hline Estrogen receptor, N (\%) & & & & 0.046 \\
\hline$<90 \%$ & $3(9)$ & $2(25)$ & $2(50)$ & \\
\hline$\geq 90 \%$ & $30(91)$ & $6(75)$ & $2(50)$ & \\
\hline Progesterone receptor, N (\%) & & & & 0.004 \\
\hline$<90 \%$ & $10(31)$ & $7(88)$ & $3(75)$ & \\
\hline$\geq 90 \%$ & $22(69)$ & $1(13)$ & $1(25)$ & \\
\hline Periductal chronic inflammation, N (\%) & & & & 0.034 \\
\hline Dense & $1(4)$ & $1(14)$ & $2(50)$ & \\
\hline Not dense & $26(96)$ & $6(86)$ & $2(50)$ & \\
\hline
\end{tabular}

Abbreviation: DCIS, ductal carcinoma in situ.

counts, comedo necrosis, estrogen receptor, progesterone receptor, and periductal chronic inflammation between three Oncotype DX DCIS Score groups were compared using Fisher's exact tests. All analyses were performed using SAS version 9.3 (Cary, NC) and a $P$ value $<0.05$ was considered significant in all analyses.

\section{Results}

The patients consisted of 46 women with DCIS. The age range of our patients was 41-84 years with a mean ( \pm s.d.) age of 64 years \pm 10 years and a median age of 66 years. The Oncotype DX DCIS Score was low in 33 patients $(72 \%)$, intermediate in 8 patients $(17 \%)$, and high in 4 patients $(9 \%)$. In one patient there was a test failure. Women with a high Oncotype DX DCIS Score were significantly younger than women with a low or intermediate score $(P=0.027$, Table 1$)$.

Size of DCIS was known in 45 of 46 patients. DCIS size ranged from 2.0 to $70 \mathrm{~mm}$ with a mean of $9 \pm 11 \mathrm{~mm}$ and a median of $6 \mathrm{~mm}$. Only 2 patients had a DCIS size over the recommended assay guideline size of $25 \mathrm{~mm}$. In one patient with a low Oncotype DX DCIS Score, DCIS size was not known. In the remaining 32 patients with a low score, the median (range) DCIS size was $6(2,31) \mathrm{mm}$. In the 8 patients with an intermediate DCIS Score, the median DCIS size was $9(3,70) \mathrm{mm}$. In the 4 patients with a high DCIS Score, the median DCIS size was 5 $(2,10) \mathrm{mm}$. There was no significant association between DCIS size and Oncotype DX DCIS Score $(P=0.193$, Table 1).

DCIS nuclear grade was known in 45 of 46 patients. DCIS nuclear grade was low in 7 patients $(16 \%)$, intermediate in 32 patients $(71 \%)$, and high in 6 patients (13\%). All 7 patients with low-nuclear grade DCIS had a low DCIS score. Of the 32 patients with intermediate-nuclear grade DCIS, 26 had a low score, 3 had an intermediate score, and 3 had a high score. For the 6 patents with high-nuclear grade DCIS, 5 had an intermediate score and 1 had a high score. Women with a low Oncotype DX DCIS score were more likely to have low-nuclear grade DCIS compared with women with an intermediate or high Oncotype DX DCIS score (21 vs 0 vs $0 \%$; $P<0.0001$, Table 1) Comedo necrosis was present in DCIS in 17 of 46 patients. Of the 33 patients with low Oncotype DX DCIS Score, 10 had comedo necrosis. Of the 8 patients with a intermediate Oncotype DX DCIS Score, 6 had comedo necrosis. Of the 4 patients with a high Oncotype DX DCIS Score, 1 had comedo necrosis. There was no significant association between comedo necrosis and Oncotype DX DCIS Score $(P=0.07$, Table 1$)$.

Estrogen receptor was positive in DCIS in 45 of 46 cases. In the one case, which was negative for estrogen receptor, the Oncotype DX DCIS Score 
was low. Of the 45 cases, which were estrogen receptor positive, estrogen was expressed in $90 \%$ or greater of the nuclei of neoplastic cells in 39 . Of these 39 cases 30 had a low DCIS score, 6 had an intermediate score, and 2 had a high score, and in one Oncotype DX DCIS Score was not known owing to test failure. Estrogen receptor was positive but present in $<90 \%$ of neoplastic cells in 7 cases of which 3 had a low score, 2 had in intermediate score, and 2 had a high score. Women with a low Oncotype DX DCIS Score were more likely to have estrogen receptor expression $\geq 90 \%$ compared with women with an intermediate or high Oncotype DX DCIS Score (91 vs 75 vs 50\%; $P=0.046$, Table 1).

Progesterone receptor was positive in 41 cases of DCIS, negative in 4 , and unknown in 1 . In the 4 cases negative for progesterone receptor, Oncotype DX DCIS Score was intermediate in 3 and low in 1. Progesterone receptor was $\geq 90 \%$ in 25 cases of which 22 had a low score, 1 had a high score, and 1 had an intermediate score, and in 1, Oncotype DX Score was unknown owing to test failure. Progesterone receptor was $<90 \%$ in 20 cases of which 10 had a low score, 7 had an intermediate score, and 3 had a high score. Women with a low Oncotype DX DCIS score were more likely to have progesterone receptor expression $\geq 90 \%$ in DCIS compared with women with an intermediate or higher Oncotype DX DCIS score (69 vs 13 vs $25 \%$; $P=0.004$, Table 1 ).

The hematoxylin and eosin-stained slide from the block sent for Oncotype DX testing was available for our review in 35 cases and mitotic figures were counted in all DCIS glands. The number of glands showing DCIS was counted and ranged from 1 to 100. The number of mitotic figures in all DCIS glands was $\leq 1$ in 21 patients, including 19 with 0 mitotic figures and 2 with 1 mitotic figure each; Of these 21 patients, 17 had a low Oncotype DX Score, 2 had an intermediate score, 1 had a high score, and 1 had a test failure. The number of mitotic figures in all glands was $>1$ (range 2-8) in 14 patients of whom 6 had a low score, 5 had an intermediate score, and 3 had a high score. Women with a low Oncotype DX DCIS Score were more likely to have a mitotic count of $\leq 1$ in DCIS compared with women with an intermediate or high Oncotype DX DCIS Score (74 vs 27 vs $25 \% ; P=0.045$, Table 1 ).

A slide was available for assessment of periductal chronic inflammation in 38 cases (H\&E slide 35 cases; immunostained slide 3 cases). A dense periductal chronic inflammatory infiltrate was present surrounding DCIS in only 4 cases (Figure 1); of these 4 cases 2 had a high Oncotype DX DCIS Score, 1 had an intermediate score, and 1 had a low score. Of the 34 cases without a dense periductal chronic inflammatory infiltrate (Figure 2), 26 had a low score, 6 had an intermediate score, and 2 had a high score. Women with a high Oncotype DX DCIS score were more likely to have dense chronic inflammatory infiltrate around DCIS glands compared with

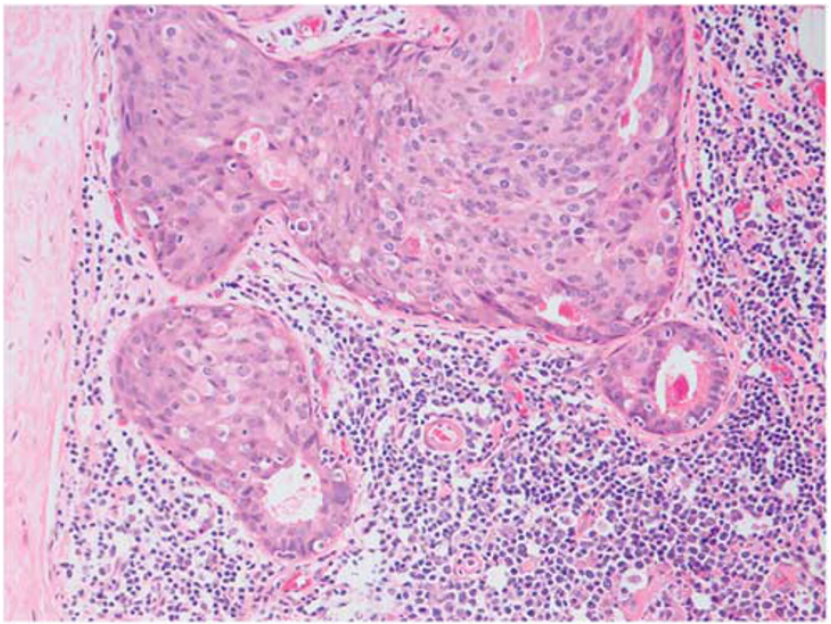

Figure 1 Ductal carcinoma in situ with dense periductal chronic inflammatory infiltrate with chronic inflammatory cells at least three layers in thickness surrounding majority of the duct circumference ( $>75 \%$ of circumference).

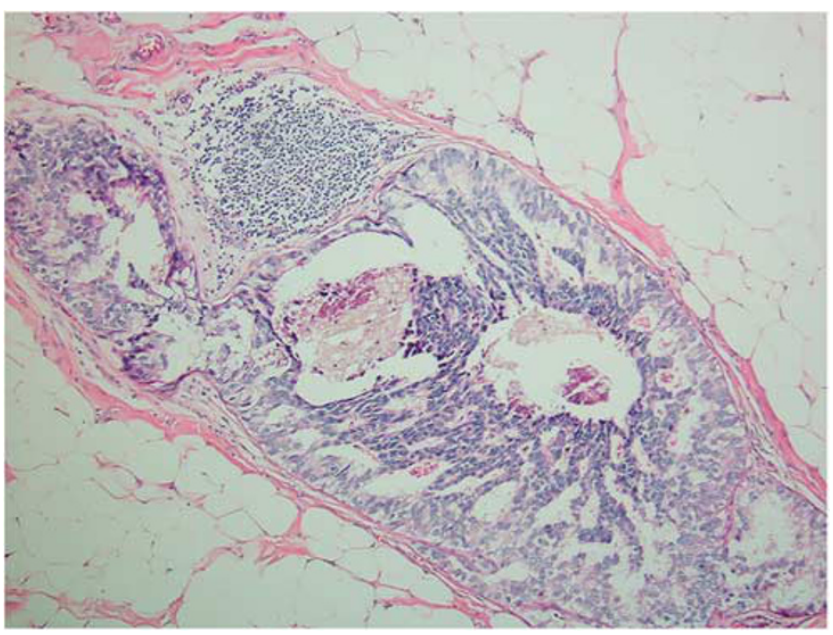

Figure 2 Ductal carcinoma in situ without dense chronic inflammatory infiltrate with chronic inflammatory cells surrounding $<75 \%$ of duct circumference.

women with an intermediate or low Oncotype DX DCIS Score (50 vs 14 vs $4 \%$; $P=0.034$, Table 1 ).

Of 46 patients, 33 patients had complete data on DCIS score, progesterone receptor expression, mitotic count. and presence or absence of dense periductal chronic inflammation. Of these 33 patients, 22 had a low Oncotype DX DCIS Score and 11 had an intermediate or high Oncotype DX DCIS Score.

Thirteen of these 33 patients had the combination of a progesterone receptor expression level $\geq 90, \leq 1$ mitotic figure, and no dense chronic inflammation around DCIS, and all 13 (100\%) had a low Oncotype DX DCIS Score. Sensitivity and specificity of using the combination of progesterone receptor $\geq 90 \%, \leq 1$ mitotic figure and no dense chronic inflammation around DCIS to identify a low Oncotype DX DCIS 
Table 2 Association between PR $\geq 90 \%$, mitotic activity $\leq 1$, and absent dense chronic inflammation around DCIS and low Oncotype DX DCIS Score

\begin{tabular}{lcc}
\hline & $\begin{array}{c}\text { Low oncotype DX DCIS } \\
\text { Score }(\mathrm{N}=22)^{\mathrm{a}}\end{array}$ & $\begin{array}{c}\text { Intermediate or high oncotype } \\
\text { DX DCIS Score }(\mathrm{N}=11)^{\mathrm{a}}\end{array}$ \\
\hline $\begin{array}{l}\text { PR } \geq 90 \% \text {, mitotic figure } \leq 1, \text { and no dense } \\
\text { chronic inflammation around DCIS }\end{array}$ & & 0.002 \\
Yes & $13(59 \%)$ & 0 \\
No & $9(41 \%)$ & $11(100 \%)$ \\
\hline
\end{tabular}

Abbreviations: DCIS, ductal carcinoma in situ; PR, progesterone receptor.

${ }^{\mathrm{a}}$ Of 45 patients with an Oncotype DX DCIS score, 33 patients had complete data on PR, mitotic count, and dense chronic inflammation around DCIS.

Table 3 Association between at least two of the following-negative PR, mitotic count $>1$, and/or dense chronic inflammation around DCIS and intermediate/high Oncotype DX DCIS Score

\begin{tabular}{|c|c|c|c|}
\hline & $\begin{array}{l}\text { Low oncotype DX DCIS } \\
\text { Score }(\mathrm{N}=22)^{\mathrm{a}}\end{array}$ & $\begin{array}{l}\text { Intermediate or high oncotype } \\
\text { DX DCIS Score }(\mathrm{N}=11)^{\mathrm{a}}\end{array}$ & $\mathrm{P}$ value \\
\hline $\begin{array}{l}\text { At least two of the following-negative } P R \text {, mitotic } \\
\text { count }>1 \text {, and dense chronic inflammation around DCIS }\end{array}$ & & & 0.0002 \\
\hline Yes & $0(0 \%)$ & $5(45 \%)$ & \\
\hline No & $22(100 \%)$ & $6(55 \%)$ & \\
\hline
\end{tabular}

${ }^{\mathrm{a}}$ Of 45 patients with an Oncotype DX DCIS Score, 33 patients had complete data on PR, mitotic count, and dense chronic inflammation around DCIS.

score was 59 and $100 \%$, respectively (Table 2). Five patients had a combination of at least two of the following-negative progesterone receptor, mitotic count $>1$, and dense chronic inflammation around DCIS, and none of these 5 patients had a low score; sensitivity and specificity of using the combination of any two of these three variables to identify cases that have a intermediate or high Oncotype DX DCIS score was 45 and $100 \%$, respectively (Table 3).

After breast conserving surgery, 14 of the 46 patients received radiation therapy including 6 of 33 with a low DCIS Score (18\%), 4 of 8 with a intermediate DCIS Score $(50 \%), 3$ of 4 with a high DCIS Score $(75 \%)$, and 1 patient with a test failure. Note the one patient with a high score who did not receive radiation therapy was advised to but refused.

\section{Discussion}

The Oncotype DX Breast Cancer Assay for DCIS is a commercially available multigene RT-PCR assay that provides an estimate of the 10-year risk of local recurrence (DCIS or invasive cancer) in patients with DCIS treated with conservative surgery without radiation therapy. ${ }^{10}$ This assay measures activity of seven genes including five proliferation genes, progesterone receptor, and GSTM-1. Both proliferative activity and progesterone receptor expression can be assessed during pathologic evaluation of DCIS. We sought to determine if pathologic features of DCIS could be used to predict Oncotype DX DCIS
Score. We found a statistically significant association between low Oncotype DX DCIS score and both progesterone receptor expression $\geq 90 \%$, and mitotic figure $\leq 1$ in DCIS. We found a statistically significant association between high Oncotype DX DCIS Score and presence of dense chronic inflammation surrounding DCIS. Furthermore, a low DCIS Score was seen in all patients with a combination of progesterone receptor $\geq 90 \%, \leq 1$ mitotic figures, and absence of dense periductal chronic inflammation around DCIS $(100 \%$ specificity). Conversely, for patients with a combination of at least two of the following, mitotic count of $>1$, negative progesterone receptor, and dense chronic inflammation around DCIS, none of these patients had a low score (100\% specificity).

In the DCIS score validation study, reported by Solin et al, ${ }^{10}$ multivariate analysis comparing traditional clinical and pathologic features of DCIS to the Oncotype DX DCIS Score demonstrated that the DCIS score was the strongest independent predictor of local recurrence risk. A study by Badve et $a 1^{13}$ correlating DCIS Score with traditional clinciopathologic features compared age, menopausal status, tamoxifen treatment, tumor size, grade, comedonecrosis, tumor type, and margin status to DCIS Score. Similar to our study they found the DCIS Score showed correlation with grade but concluded that the DCIS score provided recurrence risk information independent of these features. However, these studies did not examine and compare progesterone receptor status as an independent predictor of 
recurrence risk. In addition, mitotic activity and dense chronic inflammation around DCIS, two things we found to show statistically significant correlation with DCIS Score, were not addressed. These factors are not part of traditionally reported features of DCIS, however, they are easily assessed and our findings suggest they might add important prognostic information in DCIS. Similar to the DCIS Score, we found that not one but rather a combination of factors, including DCIS mitotic activity, progesterone receptor expression levels and presence or absence of dense chronic inflammation around DCIS, are likely to provide the greatest prognostic information.

The prognostic importance of proliferative activity, in the form of mitotic counting, is well established for invasive breast cancer. ${ }^{14}$ Assessment of mitotic activity is a key component in the Nottingham grading system for invasive breast cancer; however, a formal assessment of mitotic activity is not a mandatory part of grading for DCIS. Mitotic activity is mentioned among one of six factors to be evaluated in grading of DCIS where it is stated that mitotic activity is occasional in low-grade DCIS, intermediate in intermediate-grade DCIS, and may be frequent in high-grade DCIS; however, absence of mitotic activity does not preclude a diagnosis of high-grade DCIS. ${ }^{15}$ Research evaluating proliferative activity in DCIS and recurrence risk have focused on expression levels of the nuclear antigen Ki67, and multiple studies have shown KI67 was a predictor of local recurrence either independently or in combination with other factors. ${ }^{16}$ The Oncotype DX DCIS Score algorithm is heavily weighted toward assessment of proliferative activity with five of the seven prognostic genes assessed related to proliferation. In our study we have demonstrated a statistically significant correlation with Oncotype DX DCIS Score and mitotic activity in DCIS. This suggests that formal mitotic counting, which pathologists routinely evaluate for multiple organ systems, may have prognostic value in DCIS.

We found that the presence of dense chronic inflammation surrounding DCIS was significantly associated with a high Oncotype DX DCIS Score. Although there are no genes directly related to the immune system in the DCIS Score, research has demonstrated that there are genetic changes in the microenvironment, including within stromal fibroblasts, myoepithelium, and white blood cells, which are associated with progression from in situ to invasive disease. ${ }^{17,18} \mathrm{Ma}$ et $a 1^{19}$ reported a strong immune response signature resulting in activation of $\mathrm{T}$ cells, other leukocytes and interferon signaling present particularly around high grade DCIS. Unlike invasive cancer, where presence of a abundant tumor infiltrating lymphocytes has been linked to better prognosis, this does not appear to be the case for DCIS. ${ }^{20}$ In cases where DCIS is associated with early invasion, a dense chronic inflammatory infiltrate often surrounds these microinvasive foci. Ma and colleagues speculate that the presence of an immune response signature around high-grade DCIS may represent a phase where the cancer cells resist immune attack and instead are able to utilize the abundant cytokines produced by immune cells to facilitate invasion. ${ }^{19}$ One could also speculate that the high DCIS Score in cases with dense periductal chronic inflammation might reflect the high Ki67 index of the intermixed lymphocytes causing a falsely elevated Oncotype DCIS Score. Overall, however, our findings of a statistically significant association of a high Oncotype DX DCIS score with dense periductal chronic inflammation around DCIS suggest a role for the immune response in DCIS progression and deserves further investigation.

In our study we were able to predict a low Oncotype DX DCIS Score with $100 \%$ specificity in patients with progesterone receptor $>90 \%, \leq 1$ mitotic figure, and absence of dense chronic inflammation around DCIS. We were also able to predict with, $100 \%$ specificity as well, patients who would did not have a low DCIS Score using a combination of negative progesterone expression, mitotic count $>1$, and dense chronic inflammation around DCIS. Together these two groups represented $41 \%$ of our cohort. We could not use pathologic features to predict a intermediate vs a high DCIS Score. However, recent research by Rakovitch and colleagues, which tested the predictive ability of the DCIS Score in 713 patients with DCIS treated with breast conservation only but which included larger tumors with smaller margins, found a similar recurrence risk between patients with intermediate and high DCIS Scores (33 and $27.8 \%$, respectively). ${ }^{11}$ They suggest that it is uncertain if Genomic Health may choose to merge the intermediate- and high-risk categories.

DCIS is a complex disease and accurate determination of recurrence risk will likely be ascertained by consideration of a combination of pathologic, clinical, imaging, and molecular genetic characteristics of the tumor. The Oncotype DX DCIS test is a molecular genetic test that represents a tool to be used for assessing recurrence risk and guiding decisions about the need for radiation therapy in selected patients with DCIS. Results of molecular testing results can help us become better surgical pathologists. In this case we have correlated two pathologic features of DCIS, not routinely assessed but easily assessed, namely mitotic activity in DCIS and dense chronic inflammation around DCIS, along with progesterone receptor expression with Oncotype DX DCIS Score. Specifically, we found that in patients with DCIS showing progesterone receptor $\geq 90 \%$, $\leq 1$ mitotic figures, and absence of dense chronic inflammation around DCIS, $100 \%$ of them, representing close to one-third of our patients, had a low Oncotype DX DCIS Score. We also found that in patients with least two of the following three features in their DCIS-negative progesterone receptor, $>1$ mitotic figure, and/or dense chronic inflammation around DCIS, representing just over $10 \%$ of patients, 
none had a low score. Our finding raise the possibility that both formal mitotic counting and assessment of dense chronic around DCIS might be a valuable addition to routine pathologic assessment of DCIS and furthermore, these features in combination with progesterone receptor expression levels might serve as a surrogate for the DCIS Oncotype DX Score. In addition, when pathologists are choosing appropriate DCIS blocks for Oncotype analysis, our results suggest attention to these particular pathologic features might yield the highest score. We hope to further validate our findings on larger numbers of patients.

\section{Disclosure/conflict of interest}

$\mathrm{JF}$ is a paid consultant for Genomic Health-giving talks on the Oncotype DX testing for Breast Cancer. The remaining authors declare no conflict of interest.

\section{References}

1 Rakovitch E, Pignol JP, Chartier C et al. The management of ductal carcinoma in situ of the breast: a screened population-based analysis. Breast Cancer Res Treat 2007;101:429-438.

2 Fisher ER, Dignam J, Tan-Chiu E et al. Pathologic findings from the National Surgical Adjuvant Breast Project (NSABP) eight year update of Protocol B-17. Cancer 1999;86:429-438.

3 Wapnir I, Dignam J, Fisher B et al. Long term outcomes of invasive ipsilateral breast tumor recurrences after lumpectomy in NSABP B-17 and B-24 randomized clinical trials for DCIS. J Natl Cancer Inst 2011;103: 478-488.

4 Fisher B, Digman J, Wolmark N et al. Lumpectomy and radiation therapy for the treatment of intraductal cancer: findings from National Surgical Adjuvant Breast and Bowel Project B-17. J Clin Oncol 1998;16: 441-452.

5 Gradishar WJ, Anderson BO, Balassanian R et al. NCCN Clinical Practice Guidelines in Oncology: Breast cancer http://www.nccn.org/professions/physiciansgls/pdf/breast. pdf.Version1.2014.

6 Goodwin A, Parker S, Ghersi D et al. Post-operative radiotherapy for ductal carcinoma in situ of the breast-a systemic review of the randomized trials. Breast 2009;18:143-149.

7 Hughes L, Wang M, Page D et al. Local excision alone without irradiation for ductal carcinoma in situ of the breast: a trial of the Eastern Cooperative Oncology Group. J Clin Oncol 2009;27:5319-5324.

8 Motwani S, Goyal S, Moran M et al. Ductal carcinoma in situ treated with breast-conserving surgery and radiotherapy: a comparison with ECOG study 5194. Cancer 2011;177:1156-1162.

9 Early Breast Cancer Trialist's Collaborative Group (EBCTCG), Correa C, McGale $\mathrm{P}$ et al. Overview of the randomized trials of radiotherapy in ductal carcinoma In situ. J Natl Cancer Inst Monogr 2010;41: 162-177.

10 Solin L, Gray R, Baehner F et al. A multigene expression assay to predict local recurrence risk for ductal carcinoma in situ of the breast. J Natl Cancer Inst 2013;105:701-710.

11 Rakovitch E. Oncotype DX DCIS score predicts recurrence. Cancer Discov. 2014;5: OF3 Published online first, 23 December 2014.

12 Fang J, Wang S, Zhang S et al. Association of the glutathione s-transferase M1, T1 polymorphisms with cancer: evidence from a meta-analysis. PLOS One 2013;8:e78707.

13 Badve S, Gray R, Baehner F et al. Correlation between the DCIS score and traditional clinicopathologic features in the prospectively designed E5194 clinical validation study. J Clin Oncol 2012;30, (suppl;abstr 1005).

14 Elston C, Ellis I. Pathologic prognostic factors in breast cancer: the value of histologic grade in breast cancer: experience from a large study with long-term follow-up. Histopathology 1991;19:403-410.

15 Lester S, Bose S, Chen Y et al. Protocol for the examination of specimens from patients with DCIS of the breast http://www.cap.org/cancerprotocols Dec 2013.

16 Lari S, Kuerer N. Biologic markers in DCIS and risk of breast recurrence: a systemic review. J Cancer 2011;2: 232-261.

17 Allen M, Thomas G, Clark S et al. Altered microenvironment promotes progression of preinvasive breast cancer: myoepithelial expression of $\alpha v \beta 6$ integrin in DCIS identifies high risk patients and predicts recurrence. Clin Cancer Res 2014;20:344-357.

18 Cowell C, Weigelt B, Sakr R et al. Progression form ductal carcinoma insitu to invasive breast cancer: revisited. Mol Oncol 2013;7:859-869.

19 Ma X, Dahiya S, Richardson E et al. Gene expression profiling of the tumor microenvironment during breast cancer progression. Breast Cancer Res 2009;11:R7.

20 Salgado R, Denkert C, Demaria S et al. Harmonization of the evaluation of tumor infiltrating lymphocytes (TIL) in breast cancer: recommendations by an international TILs working group 2014. Ann Clin Oncol 2014;00:1-13. 